BULL. AUSTRAL. MATH. SOC.

VOL. $2(1970), 275-276$.

\title{
A note on types
}

\section{B. J. Gardner}

A characterization is obtained of the types $\tau$ for which $[G / G(\tau)](\tau)=0$ for all torsion-free abelian groups $G$.

In a torsion-free abelian group $G$, for any type $\tau$, the elements $x$ of $G$ with types $T(x)$ such that $T(x) \geq \tau$ form a subgroup $G(\tau)$. (We shall use the notation of [1], Chapter VII.) The aim of this note is to prove

THEOREM. Let $\tau$ be a type. Then $[G / G(\tau)](\tau)=0$ for all torsion-free abelian groups $G$ if and only if $\tau$ is the type of a height $\left(h_{1}, h_{2}, \ldots, h_{n}, \ldots\right)$ where $h_{n}$ takes only the values 0 and $\infty$.

Suppose $\tau$ is the type of a height $\left(h_{1}, h_{2}, \ldots, h_{n}, \ldots\right)$ where $h_{n}=0$ or $\infty$ for each $n$, and let $m$ be such that $h_{m}=\infty$. If for some torsion-free $G$ there is an element $g$ such that $T(g+G(\tau)) \geq \tau$, then for each positive integer $i$, there exists $g_{i} \in G$ for which $p_{m}^{i} g_{i}-g \in G(\tau)$. But then $p_{m}^{i} g_{i}-g$ is divisible by $p_{m}^{i}$, so $g$ is also. Thus $T(g) \geq \tau$, so $[G / G(\tau)](\tau)=0$.

The converse is a consequence of

PROPOSITION. Let $\tau$ be the type of a height $\left(h_{1}, h_{2}, \ldots, h_{n}, \ldots\right)$ where $0<h_{n}<\infty$ for infinitely many values of $n$. Then there is a torsion-free abelian group $G$ with the following properties:

(i) $G$ has rank 2 ,

(ii) G( $\tau$ ) has rank 1 ,

Received 21 February, 1970. 
(iii) $[G / G(\tau)](\tau)=G / G(\tau)$.

Proof. Let $M=\left\{n \mid h_{n}=\infty\right\}$. Let $\left(k_{1}, k_{2}, \ldots, k_{n}, \ldots\right)$ be the subsequence of positive finite terms of $\left(h_{1}, h_{2}, \ldots, h_{n}, \ldots\right)$ and re-label the associated primes as $q_{1}, q_{2}, \ldots$. Let $\{x, y\}$ be a basis for a two-dimensional rational vector space $\cdot V$ and $G$ the subgroup of $V$ generated by

$$
\left\{p^{-n} x, p^{-n} y, q_{n}^{-k} x, q_{n}^{-k}\left(q_{n}^{-1} x+y\right) \mid p \in M, n=1,2, \ldots\right\} .
$$

A routine argument using the linear independence of $x$ and $y$ shows that the height of $x$ is $\left(h_{1}, h_{2}, \ldots, h_{n}, \ldots\right)$, so that $T(x)=\tau$. Suppose $y$ is divisible by $q_{n}^{k}$ for some $n$. Since the same is true of $q_{n}^{-1} x+y, x$ has $q_{n}$-height $k_{n}+1$ at least, which is impossible.

Thus $T(y)<\tau$. Clearly $[x]_{*} \subseteq G(\tau)$ where $[x]_{*}$ is the smallest pure subgroup of $G$ containing $x$. If this inclusion is proper, then $G(\tau)$, being a pure subgroup, must have rank 2 and therefore coincide with $G$. But $y \notin G(\tau)$ and hence $G(\tau)=[x]_{*}$.

Let $\pi$ denote the natural homomorphism from $G$ to $G /[x]_{*} \cdot G /[x]_{*}$ is generated by

$$
\left\{p^{-n} \pi(y), q_{n}^{-k} n_{\pi(y)} \mid p \in M, n=1,2, \cdots\right\}
$$

and so is rational of type $\tau$. Thus

$$
[G / G(\tau)](\tau)=\left[G /[x]_{*}\right](\tau)=G /[x]_{*}=G / G(\tau) .
$$

\section{Reference}

[1] L. Fuchs, Abelian groups (Publishing House of the Hungarian Academy of Sciences, Budapest, 1958).

University of Tasmania, Hobart, Tasmania. 